\title{
INDEX TO VOLUME 81
}

\section{RESEARCH ANNOUNCEMENTS}

Abe, Kinetsu and Erbacher, Joseph. Nonregular contact structures on Brieskorn manifolds, 407

Akcoglu, M. A. and Sucheston, L. On weak and strong convergence of positive contractions in $L_{p}$ spaces, 105

Albertson, Michael $O$. and Hutchinson, Joan P. The maximum size of an independent set in a nonplanar graph, 554

Alfsen, Erik M. and Shultz, Frederic W. On the geometry of noncommutative spectral theory, 893

Allen, G. D. and Narcowich, F. J. On the representation and approximation of a class of operator-valued analytic functions, 410

Almgren, F. J., Jr. Existence and regularity almost everywhere of solutions to elliptic variational problems with constraints, 151

Aloff, Simon and Wallach, Nolan R. An infinite family of distinct 7-manifolds admitting positively curved Riemannian structures, 93

Anh, Nguyen Huu. Prehomogeneous vector space defined by a semisimple algebraic group, 402

Asimov, Daniel. Round handles and homotopy of nonsingular vector fields, 417

Bachmuth, S. and Mochizuki, H. Y. Automorphisms of solvable groups, 420

Becker, J. C., Casson, A. and Gottlieb, D. H. The Lefschetz number and fiber preserving maps, 425

Bender, Edward A. and Herzberg, Norman P. Some diophantine equations related to the quadratic form $a x^{2}+b y^{2}, 161$

Berenstein, Carlos A. The number of zeros of an analytic function in a cone, 213

Berger, C. A., Coburn, L. A. and Lebow, A. C*-algebras generated by commuting isometries. I, 747

Brezis, Haim and Browder, Felix E. Existence theorems for nonlinear integral equations of Hammerstein type, 73

- Maximal monotone operators in nonreflexive Banach spaces and nonlinear integral equations of Hammerstein type, 82

Browder, Felix E. See Brezis, Haim

Brown, Lawrence G. Operator algebras and algebraic $k$-theory, 1119

Bruck, Ronald E., Jr. An iterative solution of a variational inequality for certain monotone operators in Hilbert space, 890

Buck, R. C. Approximate functional complexity, 1112

Burkholder, D. L. $H^{p}$ spaces and exit times of Brownian motion, 556

Burnham, James T. Relative completions of A-Segal algebras, 125

Burns, J. A. and Herdman, T. L. Adjoint semigroup theory for a Volterra integrodifferential system, 1099

Byrnes, Christopher I. Closed algebras of smooth functions, 195

Cahn, Robert S. and Wolf Joseph A. Zeta functions and their asymptotic expansions for compact locally symmetric spaces of negative curvature, 1086

Calder, Allan. Uniformly trivial maps into spheres, 189

Cantor, Murray. Groups of diffeomorphisms of $R^{n}$ and the flow of a perfect fluid, 205

Cappell, Sylvain E. and Shaneson, Julius L. Invariants of 3-manifolds, 559

Casson, A. See Becker, J. C.

Chandler, Colston and Gibson, A. G. Invariance principle for modified wave operators, 1130

Chen, Ban-yen. Some integral inequalities of two geometric invariants, 177 
Chinburg, Ted and Hinriksen, Melvin. Sums of kth powers in the ring of polynomials with integer coefficients, 107

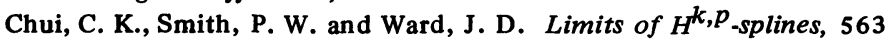

Chung, Kai Lai. Maxima in Brownian excursions, 742

Clarke, Frank H. Maximum principles without differentiability, 219

Coburn, L. A. See Berger, C. A.

Connelly, Robert. An attack on rigidity. I, II, 566

Connes, A. Classification of automorphisms of hyperfinite factors of type $\Pi_{1}$ and $\Pi_{\infty}$ and application to type III factors, 1090

Connett, William C. and Schwartz, Alan L. Unifying multiplier theorems of Hörmander, Marcinkiewicz, and Michlin type, 570

Cordoba, A. A radial multiplier and a related Kakeya maximal function, 428

Cordoba, A. and Fefferman, R. A geometric proof of the strong maximal theorem, 941

Daly, James E. The invalidity of the Calderon-Zygmund inequality for singular integrals over local fields, 896

Dash, A. T. Joint spectrum in the Calkin algebra, 1083

Davis, Donald M. and Mahowald, Mark. A strong nonimmersion theorem for $R P^{81+7}, 155$

Deodhar, Vinay V. On central extensions of rational points of algebraic groups, 573

Dhombres, Jean G. A functional characterization of Markovian linear exaves, 703

Donaldson, James A. The Cauchy problem for a first order system of abstract operator equations, 576

Dyer, Joan L. and Formanek, Edward. Complete automorphism groups, 435

Earnest, A. G. and Hsia, J. S. Springer-type theorems for spinor genera of quadratic forms, 942

Edwards, David A. and Geoghegan, Ross. The stability problem in shape and a Whitehead theorem in pro-homotopy, 438

- The Wall obstruction in shape and pro-homotopy, with applications, 919

Ehrenpreis, Leon. Hyperbolic equations and group representation, 1109

Ellis, Richard S. Concavity of magnetization for a class of even ferromagnets, 925

Erbacher, Joseph. See Abe, Kinetsu

Fefferman, R. See Cordoba, A.

Feinerman, Robert P. and Kelman, Robert B. Dual orthogonal series: an abstract approach, 733

Feldman, Jacob and Moore, Calvin C. Ergodic equivalence relations, cohomology, and von Neumann algebras, 921

Fiedorowicz, Zbigniew and Priddy, Stewart. Loop spaces and finite orthogonal groups, 700

Fife, Paul C. and McLeod, J. B. The approach of solutions of nonlinear diffusion equations to travelling wave solutions, 1076

Fine, Benjamin. The HNN and generalized free product structure of certain linear groups, 413

Formanek, Edward. See Dyer, Joan L.

Fossum, Robert M. and Griffith, Phillip A. A complete local factorial ring of dimension 4 which is not Cohen-Macaulay, 111

Frankel, Theodore. Applications of Duschek's formula to cosmology and minimal surfaces, 579

Freedman, Michael H. On the classification of Taut submanifolds, 1067

Fujii, Akio. On the distribution of the zeros of the Riemann zeta function in short intervals, 139

- A comparative study of the zeros of Dirichlet L-functions, 199

Garbanati, Dennis. Extensions of the Hasse norm theorem, 583

Geoghegan, Ross. See Edwards, David A.

Gerardin, Paul, On cuspidal representations of p-adic reductive groups, 756

Gibson, A. G. See Chandler, Colston

Ginn, S. M. and Moss, P. B. Finitely embedded modules over Noetherian rings, 709

Goldberg, Wallace. Necessary and sufficient conditions for determining a Hill's equation from its spectrum, 423

Goldston, Beth and Mewborn, A. C. A structure sheaf for a noncommutative Noetherian ring, 944 
Golitschek, M. v. Linear approximation by exponential sums on finite intervals, 443

Gottlieb, D. H. See Becker, J. C.

Greene, R. E. and Wu, H. Approximation theorems, $C^{\infty}$ convex exhaustions and manifolds of positive curvature, 101

Griffith, Phillip A. See Fossum, Robert M.

Grove, Karsten, Karcher, Hermann and Ruh, Ernst A. Group actions and curvature, 89

Grünbaum, F. Alberto, The determinant of a random matrix, 446

Hahn, Kyong T. Trigonometry on the unit ball of a complex Hilbert space, 183

Hanf, William. The Boolean algebra of logic, 587

Harrison, Jenny. On unsmoothable diffeomorphisms, 746

Hazewinkel, Michiel, A universal formal group and complex cobordism, 930

Hejhal, Dennis A. Monodromy groups for higher-order differential equations, 590

- The Selberg trace formula for congruence subgroups, 752

Hellerstein, Simon and Williamson, Jack. Derivatives of entire functions and a question of Pólya, 453

Henriksen, Melvin. See Chinburg, Ted

Herdman, T. L. See Burns, J. A.

Herzberg, Norman P. See Bender, Edward A.

Hsia, J. S. See Earnest, A. G.

Hsiang, Wu-Yi and Su, J. C. A cohomological structural theorem for topological actions of $\mathrm{Z}_{2}$-tori on spaces of $\mathrm{Z}_{2}$-cohomology type of successive fibration of projective spaces, 714

Ho, Chung-Wu. Deforming P.L. homeomorphisms on a convex 2-disk, 726

Holub, J. R. Rotundity, orthogonality, and characterizations of inner product spaces, 1087

Hönig, Chaim Samuel. Volterra-Stieljes integral equations with linear constraints and discontinuous solutions, 593

Howe, Roger and Silberger, Allan. Why any unitary principal series representation of $S L_{n}$ over a p-adic field decomposes simply, 599

Howes, F. A. Some singular perturbation problems, 602

$\mathrm{Hu}$, Men-chang. Conical distributions for rank one symmetric spaces, 98

Hulanicki, A. Commutative subalgebra of $L^{1}(G)$ associated with a subelliptic operator on a Lie group G, 121

Husseini, S. Y. The products of manifolds with the f.p.p. need not have the f.p.p., 441

Hutchinson, Joan P. See Albertson, Michael O.

Jaffee, Harris, A. Real forms of Hermitian symmetric spaces, 456

Johnson, R. Convoluteurs of $H^{p}$ spaces, 711

Josefson, Bengt. Weak sequential convergence in the dual of a Banach space does not imply norm convergence, 166

van der Kallen, Wilberd, Maazen, Hendrik and Stienstra, Jan. A presentation for some $K_{2}(n, R), 934$

Kaminker, Jerome and Schochet, Claude. Steenrod homology and operator algebras, 431

Kaplan, Aroldo and Putz, Robert. Harmonic forms and Riesz transforms for rank one symmetric spaces, 128

Karcher, Hermann. See Grove, Karsten

Kawai, Takahiro. Removable singularities of solutions of systems of linear differential equations, 461

Keene, Fredrick W. $L_{2}$-representations and a Plancherel-type theorem for parabolic subgroups, 117

Kelman, Robert B. See Feinerman, Robert P.

Kempf, George R. Images of homogeneous vector bundles and varieties of complexes, 900

Kenig, Carlos E. and Porta, Horacio. Weak continuity of Banach algebra products, 605

Kieffer, J. C. An entropy equidistribution property for a measurable partition under the action of an amenable group, 464

Kluvánek, Igor. The range of a vector measure, 609

Koschorke, Ulrich. Framefields and nondegenerate singularities, 157

Kostant, B. and Sullivan D. The Euler characteristic of an affine space form is zero, 937

Kubota, Tomio. A generalized Weil type representation and function analogous to $e^{-x^{2}}, 902$ 
Kudla, Stephen. On the R-forms of certain algebraic varieties, 471

Kulkarni, R. S. Conformal geometry in higher dimensions. I, 736

Latch, Dana May. A uniqueness theorem for homology in Cat, the category of small categories, 449

Lebow, A. See Berger, C. A.

Lebowitz, Aaron. Some degenerations of a compact Riemann surface of genus 4, 495

Lewin, Jacques and Lewin, Tekla. The group algebra of a torsion-free one-relator group can be embedded in a field, 947

Lewin, Tekla. See Lewin, Jacques

Lieberman, D. and Sernesi, E. Semicontinuity of Kodaira dimension, 459

Lin, James P. The loop space problem and its consequences, 723

Lipschutz, Seymour. The conjugacy problem and cyclic amalgamations, 114

Loebl, Richard I. and Muhly, Paul S. Reductive algebras and automorphism groups of von Neumann algebras, 759

Lozi, René. A computing method for bifurcation boughs of nonlinear eigenvalue, 1127

Maazen, Hendrick. See van der Kallen, Wilberd

Mac Camy, R. C. and Smith, R. L. Limits of solutions of Volterra integral equations, 739

Mahowald, Mark. See Davis, Donald M.

Mallet-Paret, John. Generic properties of retarded functional differential equations, 750

Matsumoto, Yukio. A 4-manifold which admits no spine, 467

McLeod, J. B. See Fife, Paul C.

Melrose, Richard B. Normal self-intersections of the characteristic variety, 939

Mewborn, A. C. See Goldston, Beth

Meyerhoff, Alan A. Proper T-maps of T-modules, 474

Miller, Haynes R., Ravenel, Douglas C. and Wilson, W. Stephen. Novikov's ext ${ }^{2}$ and the nontriviality of the gamma family, 1073

Miller, R. T. Mapping cylinder neighborhoods of some ANR's, 187

Miller, Sanford. Differential inequalities and Caratheodory functions, 79

Milman, Mario, Integral transforms of weak type between rearrangement invariant spaces, 761

Mochizuki, H. Y. See Bachmuth, S.

Moore, Calvin C. See Feldman, Jacob

Moss, P. B. See Ginn, S. M.

Muhly, Paul S. See Loebl, Richard I.

Mujica, Jorge. On the Nachbin topology in spaces of holomorphic functions, 904

Nakagami, Yoshiomi. Duality for crossed products of von Neumann algebras by locally compact groups, 1106

Narcowich, F. J. See Allen, G. D.

Nehari, Zeev. Riccati systems, 612

Newman, C. M. An extension of Khintchine's inequality, 913

Niculescu, Constantin. Absolute continuity and weak compactness, 1064

Nikol'skii, N. K. Analysis and synthesis for the point and unitary spectra. I, 169

Analysis and synthesis for the point and unitary spectra. II, 173

Oberlin, Daniel M. Multipliers of closed ideals of $L^{p}\left(D^{\infty}\right), 479$

Oliver, Robert. Fixed-points of finite group actions on contractible complexes, 482

Palmore, Julian I. Classifying relative equilibria. II, 489

Papick, Ira J. Topologically defined classes of going-down domains, 718

Paul, Jerome L. The q-regularity of lattice point paths in $R^{n}, 492$

Petrie, Ted. G-transversality, 721

Pichorides, S. K. On the conjugate of bounded functions, 143

Poénaru, V. Some invariants of generic immersions and their geometric applications, 1079 Stability of equivariant smooth maps, 1125

Porta, Horacio. See Kenig, Carlos E.

Priddy, Stewart. See Fiedorowicz, Zbigniew

Prikry, Karel. Ultrafilters and almost disjoint sets. II, 209

Ideals and powers of cardinals, 907

Prosser, Reese T. A double scale of weighted $L^{2}$ spaces, 615 
Pukanszky, L. Lie groups with completely continuous representations, 1061

Putz, Robert. See Kaplan, Aroldo

Radford, David E. The antipode of a finite-dimensional Hopf algebra over a field has finite order, 1103

Ravenel, Douglas C. See Miller, Haynes R.

Redheffer, Ray. Matrix differential equations, 485

Rochberg, Richard. Algebras of analytic functions on degenerating Riemann surfaces, 202

Rosset, Shmuel. Generic matrices, $K_{2}$, and unirational fields, 707

Roth, Alvin E. A lattice fixed-point theorem with constraints, 136

Ruh, Ernst A. See Grove, Karsten

Schochet, Claude. See Kaminker, Jerome

Schwartz, Alan L. See Connett, William C.

Sentilles, Dennis. The $L^{1}$-space of a Boolean algebra and semireflexivity of $L^{\infty}(X, \Sigma, H)$, 1096

Sernesi, E. See Lieberman, D.

Shaneson, Julius L. See Cappell, Sylvain E.

Shapiro, Daniel B. Quarterly forms and similarities, 1122

Shultz, Frederic W. See Alfsen, Erik M.

Shyr, Jih-Min. On relative class numbers of certain quadratic extensions, 500

- On the norm form of a finite Galois extension over Q, 619

Silberger, Allan. See Howe, Roger

Silberger, Allan J. Convexity for a simply connected p-adic group, 910

Smith, Larry. New infinite families in the stable homotopy of spheres, 148

Smith, P. W. See Chui, C. K.

Smith, R. L. See Mac Camy, R. C.

Sommese, Andrew J. Extension theorems for reductive group actions on compact Kaehler manifolds, 729

Sondow, Jonathan D. When is a manifold a leaf of some foliation?, 622

Stanley, Richard P. Cohen-Macaulay rings and constructible polytopes, 133

Stenger, Frank. An algorithm for the topological degree of a mapping in n-space, 179

Stienstra, Jan. See van der Kallen, Wilberd

Su, J. C. See Hsiang, Wu-Yi

Sucheston, L. See Akcoglu, M. A.

Sullivan D. See Kostant, B.

Sussman, Myron M. On uniqueness in Cauchy's problem for elliptic operators with characteristics of multiplicity greater than two, 625

Taylor, Jean E. The structure of singularities in area-related variational problems and constraints, 1093

Taylor, Joseph L. A counterexample in shape theory, 629

Toledo, Domingo and Tong, Yue Lin L. The holomorphic Lefschetz formula, 1133

Tomas, Peter A. A restriction theorem for the Fourier transform, 477

Ton-That, Tuong. On holomorphic representations of symplectic groups, 1069

Tong, Yue Lin L. See Toledo, Domingo

Torchinsky, Alberto. On a mean value inequality, 950

Trench, William F. Proof of a conjecture of Askey on orthogonal expansions with positive coefficients, 954

Wallach, Nolan R. See Aloff, Simon

Ward, J. D. See Chui, C. K.

Weight, T. Some subalgebras of $L^{\infty}(T)$ determined by their maximal ideal spaces, 192

Weiss, William A. R. A solution to the Blumberg problem, 957

Wenston, Paul R. On local solvability of linear partial differential equations not of principal type, 215

West, James E. Compact ANR's have finite type, 163

Williams, David. The $Q$-matrix problem for Markov chains, 1115

Williamson, Jack. See Hellerstein, Simon

Wilson, W. Stephen. See Miller, Haynes R.

Wolf, Joseph A. See Cahn, Robert S. 
Wu, H. See Greene, R. E.

Zafran, Misha. Measures as convolution operators on Hardy and Lipschitz spaces, 503

Zaslavsky, Thomas. Counting the faces of cut-up spaces, 916

Zettl, Anton. A constructive characterization of disconjugacy, 145

Zimmer, Robert J. Extensions of ergodic actions and generalized discrete spectrum, 633

- Distal transformation groups and fibre bundles, 959

\section{BOOK REVIEWS}

Akemann, Charles A. Review of Lectures in functional analysis and operator theory by Sterling K. Berberian; Functional analysis, a short course by Edward W. Packel; and Functional analysis by Walter Rudin, 842

Anastasio, S. See Kadison, Richard V.

Arnold, L. See Goodman, Victor

Aumann, R. J. See Isbell, J. R.

Baily, Walter L., Jr. Review of Topics in complex function theory by Carl L. Siegel, 528

Balakrishnan, A. V. See Goodman, Victor

Barwise, Jon. Review of Elementary induction on abstract structures by Y. N. Moschovakis, 1031

Bellman, R. See Papanicolaou, George C.

Bennett, Grahame. Review of Classical Banach spaces by Joram Lindenstrauss and Lior Tzafriri, 41

Berberian, Sterling, K. See Akemann, Charles A.

Berkovitz, L. D. See Fleming, W. H.

Biggs, Norman. See Wilf, Herbert S.

Boas, R. P. Review of Applied and computational complex analysis by Peter Henrici, 647

Bognár, János. See Helton, J. William

Bonsall, F. F. See Rickart, C. E.

Borho, Walter. See Hochschild, G.

Challifour, John L. Review of The $P\left(\Phi_{2}\right)$ euclidean (quantum) field theory by Barry Simon, 881

Curtis, Charles W. Review of Buildings of spherical type and finite BN-pairs by Jacques Tits, 652

Daleckii, Ju. L. See Schaffer, Juan Jorge

Devlin, Keith J. See Magidor, Menachem

Donoghue, W. F., Jr. See Karányi, Adam

Douglas, R. G. Review of Einige Klassen singularen Gleichungen by S. Prössdorf, 841

Duncan J. See Rickart, C. E.

Ekeland, I. See Morrey, Charles B., Jr.

Erdelyi, A. Review of Constructive methods for elliptic equations by R. P. Gilbert, 1036

Feinerman, Robert P. See Rivlin, Theodore J.

Fel'dman, I. A. See Widom, Harold

Fleming, W. H. Review of Optimal control theory by L. D. Berkovitz, 840

Foata, Dominique. See Whitehead, Earl Glen, Jr.

Freiman, G. A. See Gordon, Basil

Fremlin, D. H. See Gillespie, T. A.

Gabriel, Peter. See Hochschild, G.

Gilbert, R. P. See Erdelyi, A.

Gillespie, T. A. Review of Topological Riesz spaces and measure theory by D. H. Fremlin, 35

Gohberg, I. C. See Widom, Harold

Golubitsky, M. See Levine, Harold

Goodman, Victor. Review of Stochastic differential equations; theory and applications by L. Arnold; and Stochastic differential systems, I. Filtering and control, a function space approach by A. V. Balakrishnan, 837

Gordon, Basil. Review of Foundations of a structural theory of set addition by G. A. Freiman, 393 
Gruenberg, K. W. Review of Homology in group theory by Urs Stammbach, 851

Guillemin, V. See Levine, Harold

Helgason, Sigurdur. Review of Strong rigidity of locally symmetric spaces by G. D. Mostow, 509

Helton, J. William. Review of Indefinite Inner product spaces by János Bognár, 1028

Henrici, Peter. See Boas, R. P.

Hochschild, G. Review of Primideale in Einhüllenden auflösbarer Lie-algebren by Walter Borho, Peter Gabriel and Rudolf Rentschler, 39

Hofmann, Joseph E. See Weil, A.

Hofmann, K. H. See Taylor, Joseph L.

Hoover, T. B. Review of Invariant subspaces by H. Radjavi and P. Rosenthal, 31

Isbell, J. R. Review of Values of non-atomic games by R. J. Aumann and L. S. Shapley, 539

Kadison, Richard V. Review of The structure of factors by S. Anastasio and P. M. Willig, 522

Kingman, J. F. C. Review of Random sets and integral geometry by G. Matheron, 844

Kleitman, D. J. Review of Map color theorem by Gerhard Ringel, 657

Knapp, A. W. Review of Lie groups, lie algebras, and their representations by V. S. Varadarajan; and Compact Lie groups and their representations by D. P. Želobenko, 865

Korányi, Adam. Review of Monotone matrix functions and analytic continuation by W. F. Donoghue, Jr., 847

Kreìn, M. G. See Schaffer, Juan Jorge

Kuipers, L. See Tijdeman, R.

Levine, Harold. Review of Analyse differentielle by Valentin Poenaru; and Stable mapping and their singularities by M. Golubitsky and V. Guillemin, 872

Lindenstrauss, Joram. See Bennett, Grahame

van Lint, Jacobus $H$. See Wilf, Herbert S.

Magid, Andy R. Review of Conjugacy classes in algebraic groups by Robert Steinberg, 397

Magidor, Menachem. Review of Aspects of constructibility by Keith J. Devlin, 854

Matheron, G. See Kingman, J. F. C.

Mesarovic, M. D. See Mullin, Albert A.

Milnor, John W. See Spanier, E. H.

Morrey, Charles B., Jr. Review of Analyse convexe et problèmes variationelles by I. Ekeland and $R$. Teman, 857

Morrow, James A. Review of Differential analysis on complex manifolds by R. O. Wells, Jr., 660

Moschovakis, Y. N. See Barwise, Jon

Mostert, P. S. See Taylor, Joseph L.

Mostow, G. D. See Helgason, Sigurdur

Mullin, Albert A. Review of General systems theory: mathematical foundations by M. D. Mesarovic and Y. Takahara, 1042

Newman, Donald J. See Rivlin, Theodore J.

Niederreiter, H. See Tijdeman, R.

Osofsky, B. L. Review of The structure of fields by David J. Winter, 1037

Packel, Edward W. See Akemann, Charles A.

Papanicolaou, George C. Review of An introduction to invariant imbedding by R. Bellman and G. M. Wing, 1044

Poenaru, Valentin. See Levine, Harold

Prössdorf, S. See Douglas, R. G.

Rademacher, Hans. See Stark, H. M.

Radjavi, H. See Hoover, T. B.

Rentschler, Rudolf. See Hochschild, G

Rickart, C. E. Review of Complete normed algebras by F. F. Bonsall and J. Duncan, 514

Ringel, Gerhard. See Kleitman, D. J.

Rivlin, Theodore J. Review of Polynomial approximation by Robert P. Feinerman and Donald J. Newman, 28

Rosenthal, P. See Hoover, T. B. 
Rudin, Walter. See Akemann, Charles A.

Sattinger, David H. Review of Theory of branching of solutions of nonlinear equations by M. M. Vainberg and V. A. Trenogin, 886

Schaffer, Juan Jorge. Review of Stability of solutions of differential equations in Banach space by Ju. L. DaleckiI and M. G. Kreìn, 1024

Shapley, L. S. See Isbell, J. R.

Siegel, Carl L. See Baily, Walter L., Jr.

Simon, Barry. See Challifour, John L.

Spanier, E. H. Review of Characteristic classes by John W. Milnor and James D. Stasheff, 862 Stammbach, Urs. See Gruenberg, K. W.

Stark, H. M. Review of Topics in analytic number theory by Hans Rademacher, 663

Stasheff, James D. See Spanier, E. H.

Steinberg, Robert. See Magid, Andy R.

Takahara, Y. See Mullin, Albert A.

Taylor, Joseph L. Review of Cohomology theories for compact Abelian groups by K. H. Hofmann and P. S. Mostert, 1045

Teman, R. See Morrey, Charles B., Jr.

Tijdeman, R. Review of Uniform distribution of sequences by L. Kuipers and H. Niederreiter, 672

Tits, Jacques. See Curtis, Charles W.

Trenogin, V. A. See Sattinger, David H.

Tzafriri, Lior. See Bennett, Grahame

Vainberg, M. M. See Sattinger, David H.

Varadarajan, V. S. See Knapp, A. W.

Weil, A. Review of Leibniz in Paris 1672-1676, his growth to mathematical maturity by Joseph E. Hofmann, 676

Wells, R. O., Jr. See Morrow, James A.

Whitehead, Earl Glen, Jr. Review of La série génératrice exponentielle dans les problèmes d'enumeration by Dominique Foata, 885

Widom, Harold. Review of Convolution equations and projection methods for their solution by I. C. Gohberg and I. A. Fel'dman, 546

Wilf, Herbert S. Review of Algebraic graph theory by Norman Biggs; and Combinatorial theory seminar by Jacobus H. van Lint, 536

Willig, P. M. See Kadison, Richard V.

Wing, G. M. See Papanicolaou, George C.

Winter, David J. See Osofsky, B. L.

Želobenko, D. P. See Knapp, A. W.

\section{INVITED ADDRESSES}

Anderson, Donald W., 822

Arenstorf, Richard F., 1053

Atkin, A. O. L., 1055

Barnette, David W., 1060

Burgess, C. E. Embeddings of surfaces in Euclidean three-space, 795

- 698

Burkholder, Donald L., 822

Chen, Kuo Tsai, 1055

Chernoff, Herman, 1054

Chernoff, Paul R. Quasi-analytic vectors and quasi-analytic functions, 637

- 698

Conway, John H., 72

Davis, Philip J., 697

Evans, Trevor, 697

Freyd, Peter J., 1054

Gardner, Robert B., 697

Gluck, Herman. Manifolds with preassigned curvature-a survey, 313 
John, Fritz. A priori estimates, geometric effects and asymptotic behavior, 1013

- 822

Kunen, Kenneth, 1055

Harris, Theodore E., 1060

Helgason, Sigurdur, 822

Hobby, Charles R., 1053

Kac, Mark, 697

Keen, Linda, 822

Keisler, H. Jerome, 822

Lacher, R. C., 1053

Lang, Serge, 69

Lieb, Elliott H. Some convexity and subadditivity properties of entropy, 1

Mac Lane, Saunders, 822

Montgomery, Hugh L., 72

Namioka, Isaac, 1060

Ogg, A. P. Diophantine equations and modular forms, 14

O'Meara, O. Timothy, 72

Papanicolaou, George C. Asymptotic analysis of transport processes, 330

- 69

Parter, Seymour V., 699

Petryshyn, W. V. On the approximation-solvability of equations involving A-proper and Pseudo-A-proper mappings, 223

Retherford, J. R. Applications of Banach ideals of operators, 978 697

Rosenthal, Haskell P. The Banach spaces $C(K)$ and $L^{p}(\mu), 763$ 822

Rubinow, S. I. Some mathematical problems in biology, 782

- 69

Sachs, Rainer K., 822

Schmid, Wilfried, 822

Serrin, James B., 72

Shoenfield, J. R. The decision problem for recursively enumerable degrees, 973

Smith, Kennan T., 1060

Stark, H. M. The analytic theory of algebraic numbers, 961

Thurston, William P., 69

Veech, William A., 699

Wallach, Nolan R., 822

Warner, Garth W., 71

Weiss, Guido L., 1055

Wu, Hung-Hsi, 71

\section{MISCELLANEOUS}

Bylaws of the American Mathematical Society, 1137

Council and Board of Trustees, 1974 and 1975, 507

Elections to Membership, 66, 401, 552, 689, 828, 1050

Index, Volume 81, 1153

Lehrer, G. I. Errata to: Weil representations and cusp forms on unitary groups, 636

Levinson, Norman. Errata to: Zeros of derivative of Riemann's $\xi$-function, 506

Marden, Morris, Joseph L. Walsh in memoriam, 45

Meetings, Reports of,

The April Meeting in New York (Gottschalk), 69

The April Meeting in.Santa Barbara (Ross), 71

The May Council Meeting in DeKalb (Pitcher), 819

The May Meeting in DeKalb (Bateman), 71

The October Meeting in New York (Pitcher), 821

The October Meeting in Middletown (Gottschalk), 697 
The November Meeting in Nashville (Harrold), 697

The November Meeting in Los Angeles (Ross), 698

The November Meeting in Houston (Bateman), 699

The Annual Meeting in Washington, D. C. (Pitcher and Gottschalk), 822

The March Meeting in Mobile (Harrold), 1053

The March Meeting in New York (Gottschalk), 1054

The April Meeting in St. Louis (Pitcher and Bateman), 1055

The April Meeting in Monterey (Ross), 1060

The June Meeting in Pullman (Ross), 1060

Paul, Jerome L. Addendum to: The q-regularity of lattice point paths in $R^{n}, 1136$

Ponomarev, P. Corrigendum to: Class number of definite quaternary forms with nonsquare discriminant, 506

Report of the Treasurer, 834

Statement of ownership, management and circulation, 1162

Walsh, Joseph L. See Marden, Morris

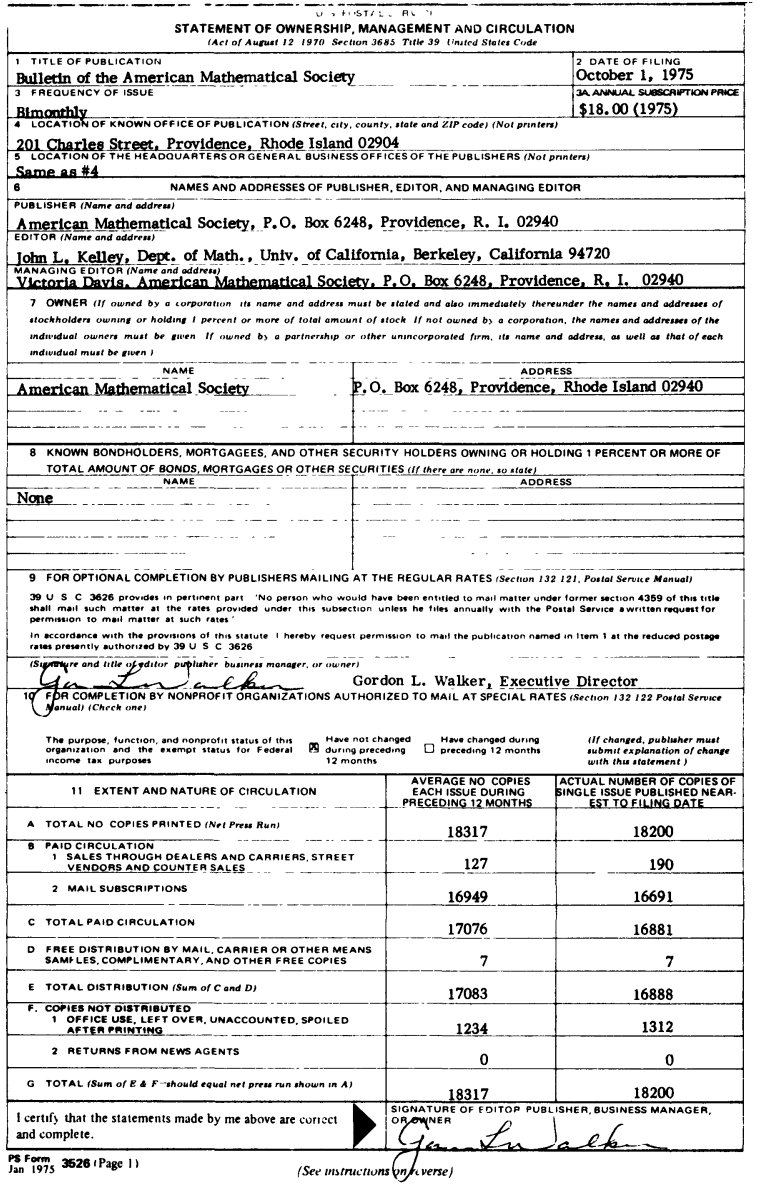

